\title{
The generalized Haar spaces and their adaptive decomposition
}

\author{
Yuri K. Demjanovich, Tatjana A.Safonova, Mikhail A.Terekhov \\ Saint Petersburg State University, Russia \\ Yuri.Demjanovich@gmail.com, y.demjanovich@spbu.ru \\ V. Belyakova \\ Immanuil Kant Baltic Federal University,Kalinigrad, Russia \\ Bich T.N.Le \\ Hue University, College of Education,Hue, Vietnam
}

Received: June 13, 2020. Revised: August 25, 2020. 2nd Revised: August 31, 2020. Accepted: September 9, 2020. Published: September 11, 2020.

\begin{abstract}
This paper is devoted to the numerical information flows and adaptive decompositions of the general Haar functions connected with them. The aim of this paper is to propose an adaptive wavelet decomposition using an adaptive compression algorithm for a flow of numerical information of length $M$ with complexity $O(M)$ and with a given precision of $\varepsilon>0$. The numerical flows are associated with irregular spline grids. This paper discusses the calibration relations, the embedding of the general Haar spaces and their wavelet decompositions. The structure of the decomposition/ reconstruction algorithms are done. The cases of the finite and the infinite flows are considered. The paper discusses various methods of adaptive Haar approximations for the flow of function values. Assuming that the values of the first derivative of the approximated function is known (exactly or approximately), the complexity of using an adaptive grid is estimated for a priori specified approximation accuracy. The number of $K$ knots in the adaptive grid determine the required amount of memory for storage of the compression results. The number of $M$ knots of the initial grid characterizes the number of operations required to obtain the adaptive compression. In the case of access to the derivative values (or their approximations) the number of digital operations is proportional to the number $M$. If it does not have access to the last ones then the number of required operations has the order of $M^{2}$ (in the general case). If additionally, the approximated flow is convex, then the number of required operations has the order of $M \log _{2} M$. In all cases the result requires the computer memory amount to be of the order of $K$.
\end{abstract}

Keywords - calibration relations, generalized Haar spaces, irregular grids, wavelet decomposition

\section{INTRODUCTION}

The processing of numerical information flows of the large $\mathrm{M}$ length is relevant now and will be relevant in the future. For a long time, there was a need to use an irregular grid for the wavelet decomposition. The flow of the initial data is often associated with an irregular initial grid (in the cases inconsistent intervals of measurement for any physical characteristics obtained by analog devices). It must be taken into account that the initial flow can have transitions from fast to slow changes and vice versa (see [1] - [39]).

As it is known, a result of the wavelet decomposition of the initial flow is two flows: one of them carries the main information (the so-called main flow), and the second contains clarifying information (the wavelet flow). The main flow is used first. This flow must satisfy conflicting requirements. On the one hand, it should have a small volume. On the other hand, it must qualitatively display the content of the initial flow. It is most convenient to associate the main flow with the grid, obtained by the enlargement of the initial grid.

The splines are a widespread apparatus for processing numerical information flows. In this regard, we mention the development of splines that satisfy additional conditions. Various types of splines are considered, including signdefined splines, the splines preserving the prescribed shape, and adaptive splines, etc. (see [7], [8], [15], [26], [27], [29]). The splines are widely used to extract basic information from noisy flows in wavelet decompositions.

Paper [2] discusses the computer complexity for the interpolation of $n$ data by the splines of order $m$ with the result $m^{2} n / 4$ arithmetical operations. The complexity of the compression of numerical flows is a basic problem for signal processing. The standard compression of flow in classical wavelet algorithms is performed by removing the components with odd knots (see [10]). In this case, there is no reason to hope for a qualitative approximation of the initial flow. The method [3] (named MARS) for flexible regression analysis of multivariate data is presented. The complexity of the model building algorithm depends significantly on the nature of the input data. Paper [4] is devoted to B-spline complexity in the case of a uniform grid. The complexity of the approximation 
is proportional to the number of data. A fast polynomial spline with prescribed properties is represented in paper [24]. In the paper, authors show that the complexity of calculating the unknown derivatives is a linear function of the length of the initial data flow. Usage of the cubic splines as the apparatus for the application for the construction of the adaptive linear filter is discussed in paper [28]. An application of parallel technology CUDA, with the usage of B-splines is demonstrated in paper [34]. The authors of all enumerated papers have a tendency to optimize spline processing.

Well-known publications do not consider the complexity of constructing adaptive wavelet decomposition algorithms for numerical information flows. In the framework of the classical approach to the wavelet expansion of Haar approximations, the investigations were carried out in [10]. The work [10] introduces irregular grids for Haar wavelets. The aforementioned work considered an irregular initial grid with the quantity of knots $M=2^{s}$. The enlarged grid obtained by deleting knots with odd numbers. The quantity of knots of the enlarged grid was $K=2^{s-1}$. However, adaptability and highquality compression with this approach should not be counted on.

The aim of this paper is to propose an adaptive wavelet decomposition using an adaptive compression algorithm for a flow of numerical information of length $\mathrm{M}$ with complexity $O(M)$ and with a given precision of $\varepsilon>0$.

The natural source of optimization is the adaptive processing for the initial data flow. Two things are important: the processing speed of such flows and the $\mathrm{K}$ length of the compression result. An additional condition is the possibility of restoring the initial flow with a given precision of $\varepsilon>0$ from the obtained compressed flow. Such property is named $\varepsilon$-compression. The most natural are adaptive algorithms that take into account the rate of initial flow change. The optimal (best) adaptive algorithm takes $O\left(M^{2}\right)$ arithmetical operations (for more exact result see XII section). If the initial flow is convex then the algorithm exists with $O\left(M \log _{2} M\right)$ arithmetical operations (see Section XIII). If the initial flow consists of the values of a function and its derivative (or derivative approximation) at the knots of a certain grid, then adaptive $\varepsilon$-compression using $O(M)$ arithmetic operations is possible (see sections II, III, IV). Although the $\varepsilon$-compressed flow will no longer be optimal, the recipient of the $\varepsilon$-comp-ressed flow can reconstruct the initial flow with $\varepsilon>0$ precision.

In the wavelet expansion the mentioned $\varepsilon$-compressed flow (called in this case the main flow) and a refinement (so-called wavelet) flow are formed. The wavelet flow has large volume. It is stored at the source (sender) and can be issued to the receiver in whole or in part on demand. The wavelet flow, together with the main flow, allows the receiver to reconstruct the initial flow exactly. This is the value of the wavelet decomposition. However, with the classical approach, the construction of an adaptive wavelet decomposition is not possible. The nonclassical approach developed in this work leads to both adaptive $\varepsilon$-compression and adaptive wavelets (see $\mathrm{V}$-- XI sections).

The proposed work was performed in the framework of the nonclassical theory of wavelets (see [19], [30], [37] - [39]).
Here the initial grid is irregular, the quantity of its knots is arbitrary, and the main grid can be any subset of the initial grid. The enlargement of the grid is carried out gradually by removing one knot after another. This can be useful when implementing an adaptive algorithm for processing the initial flow, coming in real time.

First, we consider the auxiliary function $f$ defined at the knots of a grid called the initial grid. It is shown that this grid can be enlarged in such a way that certain properties will be satisfied, depending on the function $f$ and the number $\varepsilon>0$. The resulting grid is called an adaptive grid. It is shown that the computational complexity of constructing this grid is directly proportional to the number of knots in the initial grid. Adaptive approximation (adaptive compression) is closely related to the selection of the adaptive spline grid. The last one may be defined by the values of the function itself, and also by the values of its derivative.

We estimate the complexity of the algorithm for approximating functions of classes $C^{1}$ using the interpolation by general Haars' functions, both on a uniform and (generally speaking) non-uniform adaptive grid. Assuming that the values of the first derivative of the approximated function is known (exactly or approximately), the complexity of using an adaptive grid is estimated for a priori specified approximation accuracy. It is established that the complexity is directly proportional to the number of knots in the initial grid. The number of $K$ knots in this grid determines the required amount of memory for the storage of compression results, and the number of $M$ knots of the initial grid characterizes the number of operations required to obtain adaptive compression. Due to the fact that the implementation of a uniform grid contained in the initial grid is not always possible, a pseudo-uniform grid is introduced. The numbers of knots of adaptive and pseudouniform grids are compared to the same approximation, and the asymptotic behavior of their relationship is established. If the values of the derivative of the approximated function are not known, another algorithm for constructing an adaptive grid is proposed, the complexity of which is proportional to $M^{2}$, where $M$ is the number of knots of the initial grid. Finally, if the approximated function is convex, then a method is proposed whose complexity is $M \log _{2} M$.

\section{SOME AUXILIARY ASSERTIONS}

\section{A. Adaptive grid}

Consider a positive continuous function $f(t)$,

$$
f \in C[a, b], \quad f(t)>0 \quad \forall t \in[a, b] .
$$

Let $\varepsilon$ be a positive value. We discuss a grid

$$
\tilde{X}(f, \varepsilon): \quad a=\tilde{x}_{0}<\tilde{x}_{1}<\cdots<\tilde{x}_{K} \leq \tilde{x}_{K+1}=b
$$

such that

$$
\begin{gathered}
\max _{t \in\left[\tilde{x}_{s}, \tilde{x}_{s+1}\right]} f(t)\left(\tilde{x}_{s+1}-\tilde{x}_{s}\right)=\varepsilon \quad \forall s \in\{0,1, \ldots, K-1\} \\
\max _{t \in\left[\tilde{x}_{K}, \tilde{x}_{K+1}\right]} f(t)\left(\tilde{x}_{K+1}-\tilde{x}_{K}\right) \leq \varepsilon .
\end{gathered}
$$

Grid (2)-(4) is named an adaptive grid for the function $f$.

The next assertion holds.

Lemma 1. If relations (1) are right, then for arbitrary

$$
\varepsilon \in\left(0, \varepsilon_{0}\right), \quad \varepsilon_{0}=(b-a) \max _{t \in[a, b]} f(t),
$$


a natural number $K=K(f, \varepsilon)$ and a grid

$$
\tilde{X}(f, \varepsilon)=\left\{\tilde{x}_{i}(f, \varepsilon)\right\}_{i \in\{0,1, \ldots, K, K+1\}}
$$

exist such that the properties (3)-(4) are fulfilled. The number $K(f, \varepsilon)$ is unique.

Proof. The lemma is proved by mathematical induction over the number of knots.

I. The induction base is set as follows. Let the variable $\tau$ increase from $a=\tilde{x}_{0}$ to $b$. Then, in view of the assumptions (1) the function $\varphi_{0}(\tau)=\max _{t \in\left[\tilde{x}_{0}, \tau\right]} f(t)\left(\tau-\tilde{x}_{0}\right) \quad$ is strictly increasing. When changing $\tau$ from $\tilde{x}_{0}$ to $b$ the function $\varphi_{0}(\tau)$ increases from 0 to $\max _{t \in[a, b]} f(t)(b-a)$. By condition (5) the unique point $\tau_{1} \in[a, b]$ exists that $\max _{t \in\left[a, \tau_{1}\right]} f(t)\left(\tau_{1}-a\right)=\varepsilon$. By definition we put $\tilde{x}_{1}=\tau_{1}$. The induction base is set.

II.We suppose that knots $\tilde{x}_{1}, \tilde{x}_{2}, \ldots, \tilde{x}_{s}$ of the grid $X$ have been defined. If $\tilde{x}_{s}=b$, then we put $K=s-1$. In this case the construction of the grid $\tilde{X}(f, \varepsilon)$ is completed. Otherwise, $\tilde{x}_{s}<b$ the construction of the grid continues.

Consider a function $\varphi_{s}(\tau)=\max _{t \in\left[\tilde{x}_{s}, \tau\right]} f(t)\left(\tau-\tilde{x}_{s}\right)$. If $\tau$ changes from $\tilde{x}_{s}$ to $b$ then the function $\varphi_{s}(\tau)$ increases from $m_{s}=\max _{t \in\left[\tilde{x}_{s}, b\right]} f(t)\left(b-\tilde{x}_{s}\right)$. If $\varepsilon<m_{s}$ then $\tau_{s+1}<b$ exists such that $\varphi_{s}\left(\tau_{s+1}\right)=\varepsilon$. In this case we put $\tilde{x}_{s+1}=\tau_{s+1}$. If $\varepsilon \geq m_{s}$ then we put $K=s-1$ and $\tilde{x}_{s+1}=b$. Taking into account the relation

$\tilde{x}_{s+1}-\tilde{x}_{s}=\frac{\varepsilon}{\max _{t \in\left[\tilde{x}_{S}, \tilde{x}_{S+1}\right]} f(t)} \geq \frac{\varepsilon}{\max _{t \in[a, b] t \in[a, b]} f(t)}$,

we conclude that the mentioned process is finite.

Induction has finished. It is evident that properties (3) - (4) are fulfilled.

The grid $\tilde{X}$ is called an adaptive grid.

It is possible to discuss an initial fine grid

$$
\Xi: \quad \ldots<\xi_{-2}<\xi_{-1}<\xi_{0}<\xi_{1}<\xi_{2}<\cdots
$$

and consider the function $f(t)$ defined on a grid segment

$\llbracket a, b \rrbracket=\left\{a=\xi_{0}, \xi_{1}, \ldots, \xi_{M+1}=b\right\}$. There is analog of the Lemma 1 , in which $\tilde{X} \subset \llbracket a, b \rrbracket$.

It is evident that the next assertions are true.

Lemma 2. If conditions (1)-(4) are true and $\varepsilon \rightarrow+0$ then the integer function $K(f, \varepsilon)$ increases. The next relations

$\left.\lim _{\varepsilon \rightarrow 0 s \in\{0,1, \ldots, K\}} \max _{s+1}-\tilde{x}_{s}\right)=0, \lim _{\varepsilon \rightarrow 0} K(f, \varepsilon)=+\infty$ are right.

Lemma 3. Under conditions (1)-(4) the relation

$\lim _{\varepsilon^{\prime} \rightarrow 0 \varepsilon^{\prime} \rightarrow 0} K\left(f, \varepsilon^{\prime}\right) \varepsilon^{\prime}=\int_{a}^{b} f(t) d t \leq K(f, \varepsilon)+1$

fulfilled.

Summing (3) - (4), we have

$$
\begin{array}{r}
K \varepsilon=\sum_{s=0}^{K-1} \max _{t \in\left[\tilde{x}_{s}, \tilde{x}_{s+1}\right]} f(t)\left(\tilde{x}_{s+1}-\tilde{x}_{s}\right) \leq \\
\leq \sum_{s=0}^{K} \max _{t \in\left[\tilde{x}_{s}, \tilde{x}_{s+1}\right]} f(t)\left(\tilde{x}_{s+1}-\tilde{x}_{s}\right) \leq(K+1) \varepsilon .
\end{array}
$$

It is easy to see that the proof of Lemma 1 is actually an algorithm for construction of the grid $\tilde{X}$. Consider the question of the complexity of the calculations in this algorithm.

Let $N_{\text {add }}=N_{\text {add }}(f)$ and $N_{m u l}=N_{m u l}(f)$ be a number of additive and a number of multiplicative operations accordingly, as well as $N(f)$ and $N_{\text {comp }}=N_{\text {comp }}(f)$ be a number of calculations of the function $f(t)$ and a number of comparisons.

Lemma 4. The algorithm for the construction of the adaptive grid $\tilde{X}$ as the next properties

$$
\begin{gathered}
N(f)=N_{a d d}=N_{\text {mul }}=K+M+2, \\
N_{\text {comp }}=2(K+M+2) .
\end{gathered}
$$

Proof. The resulting grid has the form $\tilde{X}=\tilde{X}(f, \varepsilon)$ :

$$
\begin{gathered}
a=\tilde{x}_{0}=\xi_{0}<\tilde{x}_{1}=\xi_{p_{1}}<\cdots \\
\ldots<\tilde{x}_{K}=\xi_{p_{K}}<\tilde{x}_{K+1}=\xi_{p_{K}+1}=b,
\end{gathered}
$$

where $p_{K+1}=M+1$. At the $s$-th step of this algorithm, we move from the $\operatorname{knot} \tilde{x}_{s}$ o the knot $\tilde{x}_{s+1}$.

Suppose that $\tilde{x}_{s}=\xi_{p_{s}}, \tilde{x}_{s+1}=\xi_{p_{s+1}}$. It is not difficult to see that with the mentioned transition it is required 1) to calculate $p_{s+1}-p_{s}+1$ times the function $f(t)$ (at points $\xi_{p_{s}+1}$, $\left.\left.\xi_{p_{s}+2, \ldots,}, \xi_{s_{s+1}+1}\right), 2\right)$ find the maximum of two numbers $p_{s+1}-p_{s}+1$ times (by searching maxima $\max f(t)$ ), 3) execute $p_{s+1}-p_{s}+1$ additive operations, 4) execute $p_{s+1}-p_{s}+1$ multiplicative operations, 5) compare the result with $\varepsilon$ also $p_{s+1}-p_{s}+1$ times. Since $s$ should be changed from 0 to $K$, then the total number of $N(f)$ calculations of the function $f$ is

$$
N(f)=\sum_{s=0}^{K}\left(p_{s+1}-p_{s}+1\right)=K+1+p_{K+1}-p_{0} .
$$

Since, in accordance with (2) and (9), $p_{0}=0, p_{K+1}=M+1$, then as a result, we get $N(f)=K+M+2$. The same way we find the number $N_{\text {add }}$ additive and the number $N_{m u l}$ multiplicative operations, as well as the number $N_{\text {comp }}$ comparisons. So we get (8). This completes the proof.

\section{Pseudo-equidistant grid}

Let $J_{m}$ be a set $\{0,1,2, \ldots, m\}$. Subset

$$
\bar{X}: \quad a=\bar{x}_{0}<\bar{x}_{1}<\cdots<\bar{x}_{N+1}=b
$$

of the grid segment $\llbracket a, b \rrbracket$ is called pseudo-equidistant grid with grid width $h>0$, if the next relations hold

$$
\bar{x}_{j+1}-\bar{x}_{j}-\tau \leq \square<\bar{x}_{j+1}-\bar{x}_{j}+\tau, \quad j \in J_{N},
$$

where $\tau=\max _{j \in J_{M}}\left(\xi_{j+1}-\xi_{j}\right)$.

Suppose that the condition

$$
\tau \leq h<b-a .
$$

is fulfilled.

Let $q$ be a real value. The expression $\lfloor q\rfloor$ means an integer number $k_{1}$ with the property $0 \leq q-k_{1}<1$. Analogously $|q|$ means an integer $k_{2}$ with the property $0 \leq k_{2}-q<1$.

\section{B. Digital complexity}


By definition we put $N=\left\lfloor\frac{b-a}{\square}\right\rfloor$. For $j \in J_{N}$ we find $s \in J_{M}$ such that the inequality

$$
\xi_{s} \leq j h<\xi_{s+1}
$$

is right. By supposition (12) the unique number $s=s(j)$ exists. Let us discuss

$$
\bar{x}_{j}=\xi_{s(j)} \quad \forall j \in J_{N}
$$

Lemma 5. The next relation holds

$$
\square-\tau<\bar{x}_{j+1}-\bar{x}_{j} \leq \square+\tau \text {. }
$$

Proof. We assume that $\xi_{s} \leq j \square<\xi_{s+1}, \xi_{p} \leq(j+1) \square<$ $\xi_{p_{+1}}$. By supposition (12) we have $s<p$.If we put $\eta=\xi_{s_{+1}}-j \square$,

$$
\xi_{s+1}=j h+\eta, \xi_{p}=(j+1) h-\delta .
$$

By (16) we deduce

$0<\eta \leq \xi_{s+1}-\xi_{s}, 0 \leq \delta<\xi_{p_{+1}}-\xi_{p}$.

According to formulas (13) - (14) we define

$$
\bar{x}_{j}=\xi_{s}, \quad \bar{x}_{j+1}=\xi_{p} .
$$

Taking into account formula (15), we have $\xi_{s}=\xi_{s+1}-$ $\left(\xi_{s+1}-\xi_{s}\right)=j \square+\eta-\left(\xi_{s+1}-\xi_{s}\right)$. Thus we deduce

$$
\begin{gathered}
\bar{x}_{j+1}-\bar{x}_{j}=\xi_{p}-\xi_{s}=(j+1) \square-\delta-j \square+\eta- \\
\left(\xi_{s+1}-\xi_{s}\right)=\square-\delta-\eta+\left(\xi_{s+1}-\right.
\end{gathered}
$$

$\left.\xi_{s}\right)$. (18)

By relations (18) and (17) we obtain (15). This completes the proof.

Relation (11) follows from (15), therefore the obtained grid is pseudo-equidistant.

Remark 1. If the initial grid is equidistant, $\xi_{s}=s \tau$,

$\tau=\frac{b-a}{\bar{M}+1}, s=0,1, \ldots, \bar{M}$ then inequality(13) has the form $s \tau \leq j h<(s+1) \tau$. The last one is equivalent to the relation $s<\frac{j \square}{\tau}<(s+1)$. Therefore we can put $s=\left\lfloor j \frac{\square}{\tau}\right\rfloor$. Thus we have $\left.\bar{x}_{j}=\xi_{[j}\right]_{\bar{\tau}}$. Let $\varepsilon>0$ be a positive value. We suppose that

$$
N=\left\lfloor\frac{\|f\|_{C[a, b]}(b-a)}{\varepsilon}\right\rfloor>3 .
$$

Then

$$
\frac{\|f\|_{C[a, b]}(b-a)}{\varepsilon}-1<N \leq \frac{\|f\|_{C[a, b]}(b-a)}{\varepsilon} .
$$

The last inequality is equivalent to the relation

$$
\|f\|_{C[a, b]}(b-a)-\varepsilon<N_{\varepsilon} \leq\|f\|_{C[a, b]}(b-a) .
$$

We suppose that

$$
\frac{b-a}{N+1}>2 \tau
$$

Choosing the value $h$ according to the formula

$$
\square=\frac{b-a}{N+1}-\tau,
$$

We see that by condition (19) relation (12) is fulfilled. By (20) and (23) we have $N+1>\|f\|_{C[a, b]}(b-a) / \varepsilon$ and

$$
\square+\tau<\frac{b-a}{\|f\|_{C[a, b]}(b-a) / \varepsilon}=\frac{\varepsilon}{\|f\|_{C[a, b]}} .
$$

By (24) we have

$$
\begin{gathered}
\|f\|_{C[a, b]}\left(\bar{x}_{s+1}-\bar{x}_{s}\right) \leq\|f\|_{C[a, b]}\left(\bar{x}_{s+1}-\bar{x}_{s}+\tau\right) \leq \\
\leq\|f\|_{C[a, b]}(\square+\tau) \leq \varepsilon .
\end{gathered}
$$

Thus

$$
\max _{t \in\left[\bar{x}_{s}, \bar{x}_{s+1}\right]} f(t)\left(\bar{x}_{s+1}-\bar{x}_{s}\right) \leq \varepsilon \quad \forall s \in J_{N} .
$$

The previous arguments prove the following statement.

Theorem 1. If relations (19), (22) are right then the pseudo-equidistant grid (10) exists and properties (21), (25) are fulfilled.

$D_{\delta}$ Some $_{=}$assertions $\xi_{p}$ then we have

Lemma 6. If conditions (1) - (4), (19) - (23) are right then the next inequality is fulfilled:

$$
\begin{aligned}
& \frac{\sum_{S=0}^{K} \max _{t \in\left[\tilde{x}_{S}, \tilde{x}_{S+1}\right]} f(t)\left(\tilde{x}_{s+1}-\tilde{x}_{s}\right)-\varepsilon}{(b-a)\|f\|_{C[a, b]}(b-a)\|f\|_{C[a, b]}} \leq \\
& \leq \frac{K(f, \varepsilon) K(f, \varepsilon)}{N(f, \varepsilon) N(f, \varepsilon)} \\
& \leq \frac{\sum_{S=0}^{K} \max _{t \in\left[\tilde{x}_{s}, \tilde{x}_{s+1}\right]} f(t)\left(\tilde{x}_{s+1}-\tilde{x}_{s}\right)}{(b-a)\|f\|_{C[a, b]}-\varepsilon(b-a)\|f\|_{C[a, b]}-\varepsilon}
\end{aligned}
$$

Proof. By (7) we have

$$
\begin{aligned}
& \sum_{s=0}^{K} \max _{t \in\left[\tilde{x}_{s}, \tilde{x}_{s+1}\right]} f(t)\left(\tilde{x}_{s+1}-\tilde{x}_{s}\right)-\varepsilon \leq \\
\leq & K \varepsilon=\sum_{s=0}^{K-1} \max _{t \in\left[\tilde{x}_{s}, \tilde{x}_{s+1}\right]} f(t)\left(\tilde{x}_{s+1}-\tilde{x}_{s}\right) .
\end{aligned}
$$

Using relations (19) - (22), we deduce

$$
(b-a) \max _{t \in[a, b]} f(t)-\varepsilon \leq N \varepsilon \leq(b-a) \max _{t \in[a, b]} f(t) \text {. }
$$

By (27) - (28) we obtain (26).

Theorem 2. If the conditions of Lemma 5 are true then the relation

$$
\lim _{\varepsilon \rightarrow+0} \frac{K(f, \varepsilon) K(f, \varepsilon)}{N(f, \varepsilon) N(f, \varepsilon)}=\frac{\frac{1}{b-a} \int_{a}^{b} f(t) d t}{\|f\|_{C[a, b]}}
$$

is right.

Proof. Passaging to the limit in (26) under condition $\varepsilon \rightarrow$ +0 , we obtain relation (29).

III. ON THE QUANTITY OF THE KNOTS FOR THE ADAPTIVE GRID Consider a grid

$$
\hat{X}: \quad a=\hat{x}_{0}<\hat{x}_{1}<\cdots<\hat{x}_{K} \leq \hat{x}_{K+1}=b .
$$

Suppose $u \in C[a, b]$. Let $\tilde{u}$ be a piecewise linear function

$$
\tilde{u}(t)=u\left(\hat{x}_{i}\right)+\frac{u\left(\hat{x}_{i-1}\right)-u\left(\hat{x}_{i}\right)}{\hat{x}_{i+1}-\hat{x}_{i}}\left(t-\hat{x}_{i}\right), \quad t \in\left[\hat{x}_{i}, \hat{x}_{i+1}\right] .
$$

The next assertion is evident.

Lemma 7. Suppose $t \in\left[\hat{x}_{i}, \hat{x}_{i+1}\right]$. If $u \in C^{1}\left[\hat{x}_{i}, \hat{x}_{i+1}\right]$ then the inequality

$$
|u(t)-\tilde{u}(t)| \leq\left(\hat{x}_{i+1}-\hat{x}_{i}\right) \max _{\xi \in\left[\hat{x}_{i}, \hat{x}_{i+1}\right]}\left|u^{\prime(\xi)} u^{\prime}(\xi)\right|
$$


is fulfilled.

Theorem 3. Let $u \in C^{1}[a, b]$. Suppose the condition

$$
u^{\prime}(t) \neq 0 \quad \forall t \in[a, b]
$$

is true. If for $\eta>0$ the grid (30) coincides with the grid $\tilde{X}\left(\left|u^{\prime}\right|, \eta\right)$ then

1) the quantity $K_{u}^{\prime}(\eta)=K\left(\left|u^{\prime}\right|, \eta\right)$ of knots satisfies the relations

$$
\lim _{\eta^{\prime} \rightarrow+0} K\left(\left|u^{\prime}\right|, \eta^{\prime}\right)=\int_{a}^{b}\left|u^{\prime}(t)\right| d t \leq K\left(\left|u^{\prime}\right|, \eta^{\prime}\right)+1
$$

2) the inequality

$$
|u(t)-\tilde{u}(t)| \leq \eta \quad \forall t \in[a, b]
$$

is fulfilled.

Proof. Assuming $\hat{X}=\tilde{X}\left(\left|u^{\prime}\right|, \eta\right)$, we apply Lemma 3. As a result we have relation (32). Inequality (33) follows from Lemma 7 and formulas (3) - (4) for $f=\left|u^{\prime}\right|$ and $\varepsilon=\eta$.

\section{ON THE QUANTITY OF THE KNOTS IN THE}

\section{CASE OF AN EQUIDISTANT GRID}

By the value $\eta>0$ we construct an equidistant grid $\bar{X}\left(\left|u^{\prime}\right|, \eta\right)$ with the step $h=(b-a) / N\left(\left|u^{\prime}\right|, \eta\right)$, where $N\left(\left|u^{\prime}\right|, \eta\right)$ is the quantity of knots for the mentioned grid.

Theorem 4. Consider $u \in C^{1}[a, b], \eta>0$. If the grid $\hat{X}$ coincides with the grid $\bar{X}\left(\left|u^{\prime}\right|, \eta\right)$ then

1) the number $N_{u}^{\prime}(\eta)=N\left(\left|u^{\prime}\right|, \eta\right)$ of knots satisfies to the relation

2) the inequality

$$
N\left(\left|u^{\prime}\right|, \eta\right)=(b-a) \max _{t \in[a, b]}\left|u^{\prime}(t)\right| / \eta,
$$

$$
|u(t)-\tilde{u}(t)| \leq \eta \quad \forall t \in[a, b]
$$

is true.

Proof. Setting $\hat{X}=\bar{X}\left(\left|u^{\prime}\right|, \eta\right)$, we apply formula (19). As a result we obtain relation (34). Inequality (35) follows from definition of the grid $\hat{X}\left(\left|u^{\prime}\right|, \eta\right)$ (Lemma 7 and formulas (19) (22), (25) for $f=\left|u^{\prime}\right|$ and $\varepsilon=\eta$ ).

\section{COORDINATE SPLINES OF THE ZERO ORDER}

Let $(\alpha, \beta)$ be an interval of the $R^{1}$. Consider a grid

$$
\Xi: \quad \ldots<\xi_{-2}<\xi_{-1}<\xi_{0}<\xi_{1}<\xi_{2} \ldots
$$

with the properties $\lim _{j \rightarrow-\infty} \xi_{j}=\alpha, \lim _{j \rightarrow+\infty} \xi_{j}=\beta$.

Let $\omega_{j}(t)$ be a function defined by the relation

$$
\omega_{j}(t)=\left\{\begin{array}{l}
1 \text { for } t \in\left[\xi_{j}, \xi_{j+1}\right) \\
0 \text { for } t \notin\left[\xi_{j}, \xi_{j+1}\right) .
\end{array}\right.
$$

It is evident that the system of functions $\left\{\omega_{j}(t)\right\}_{j \in Z}$ is a linear independent system. The functions $\omega_{j}(t)$ are called a coordinate splines of the zero order. If the grid (36) is equidistant (and consequently, $\alpha=-\infty, \beta=+\infty$ ) then the functions $\omega_{j}$ defined above are the Haar functions.

By definition, put $S_{j}=\left[\xi_{j}, \xi_{j+1}\right]$. It is clear to see that $\operatorname{supp} \omega_{j}$ $=S_{j}$. If $t \in(\alpha, \beta)$ then the linear combination $u(t)=$ $\sum_{j \in Z} c_{j} \omega_{j}(t)$ is sensible because for fixed $t \in\left[\xi_{i}, \xi_{i+1}\right)$ the sum has only one nonzero summand, $u(t)=c_{i} \omega_{i}(t)$. Consider the linear space $S_{0}(\Xi)$ defined by the relation

$S_{0}(\Xi)=C l_{p}\left\{\tilde{u}(t) \mid \tilde{u}(t)=\sum_{j \in Z} c_{j} \omega_{j}(t) \quad t \in(\alpha, \beta)\right\}, \forall c_{j} \in$ $\square^{1}$; here a symbol $C l_{p}$ designates the closure in the point-wise topology. The space $\mathrm{S}_{0}(\Xi)$ is called the spline space of the zero order for the grid $\Xi$. The elements of this space are named the splines of the zero order. It is evident that the setting of the grid defines the space $S_{0}(\Xi)$ uniquely. We observe that the splines are defined for $t \in(\alpha, \beta)$.

\section{THE SPACES}

VI. GRID ENLARGEMENT AND EMBEDDING OF

Let $k$ be a fixed number, $k \in Z$. We discuss the numbers

$$
\begin{gathered}
\tilde{\xi}_{j}=\xi_{j} \text { for } j \leq k, \text { and } \\
\tilde{\xi}_{j}=\xi_{j+1} \text { for } j \geq k+1, \\
\eta=\xi_{k+1} .
\end{gathered}
$$

By (38) we introduce a new grid

$$
\tilde{\Xi}: \ldots<\tilde{\xi}_{-2}<\tilde{\xi}_{-1}<\tilde{\xi}_{0}<\tilde{\xi}_{1}<\tilde{\xi}_{2} \ldots
$$

Let $\widetilde{\omega}_{j}(t)$ be a piecewise constant function defined by the relation

$$
\widetilde{\omega}_{j}(t)=\left\{\begin{array}{l}
1 \text { for } t \in\left[\tilde{\xi}_{j}, \tilde{\xi}_{j+1}\right) \\
0 \text { for } t \notin\left[\tilde{\xi}_{j}, \tilde{\xi}_{j+1}\right) .
\end{array}\right.
$$

It is clear that $\left.\left[\tilde{\xi}_{k}, \tilde{\xi}_{k+1}\right)=\xi_{k}, \xi_{k+1}\right) \cup$

$\left.\xi_{k+1}, \xi_{k+2}\right)$. $\left.\left.\left[\tilde{\xi}_{k}, \tilde{\xi}_{k+1}\right)=\xi_{k}, \xi_{k+1}\right) \cup \xi_{k_{+1}}, \xi_{k_{+2}}\right)$.Taking into account definition (37) and (39), we have calibration relations

$\widetilde{\omega}_{k}(t)=\omega_{k}(t)+\omega_{k_{+1}}(t)$,

$$
\widetilde{\omega}_{j}(t)=\left\{\begin{array}{c}
\omega_{j}(t) \text { for } j \leq k-1 \\
\omega_{j+1}(t) \text { for } j \geq k+1
\end{array} \quad \forall t \in(\alpha, \beta) .\right.
$$

The calibration relations can be written in the general form

$$
\widetilde{\omega}_{i}=\sum_{j \in Z} p_{i j} \omega_{j}
$$

where $p_{s j}=\delta_{s, j}$ for $s \leq k-1, p_{s j}=\delta_{k, j}+\delta_{k, j-1}$ for $s=k$

$$
p_{s j}=\delta_{s, j_{-1}} \text { for } s \geq k+1 \text {, for all } s, j \in Z .
$$

Here $\delta_{i, j}$ is the Kronecker symbol. Let $\mathfrak{P}$ be a matrix $\left(p_{i j}\right)_{i, j \in Z}$. By definition put $\tilde{S}_{j}=\left[\tilde{\xi}_{j}, \tilde{\xi}_{j+1}\right] . \tilde{S}_{j}=\left[\tilde{\xi}_{j}, \tilde{\xi}_{j+1}\right]$. Consider a linear space

$S_{0}(\widetilde{\Xi}) C l_{p}=\tilde{u}(t) \mid \tilde{u}(t)=\sum_{j \in Z} c_{j} \widetilde{\omega}_{j}(t) \forall c_{j} \in R^{1}, t \in(\alpha, \beta)$.

Theorem 5. The space $S_{0}(\tilde{\Xi})$ is the subspace of the space $S_{0}(\Xi)$.

Proof. The proof follows from formula (42). 


\section{BIORTHOGONAL SYSTEMS OF FUNCTIONALS}

In the space $S_{0}(\Xi) S_{0}(\Xi)$ we consider the linear functionals $g_{i}$ and $\tilde{g}_{i}$ defined by the formulas

$$
\left\langle g_{i}, u\right\rangle=u\left(\xi_{i}\right),\left\langle\tilde{g}_{i}, u\right\rangle=u\left(\tilde{\xi}_{i}\right) \forall u \in S_{0}(\Xi) \forall i \in Z .
$$

Theorem 6. The next assertions are right:

1) the system $\left\{g_{i}\right\}_{i \in Z}$ of the functionals are biorthogonal to the system $\left\{\omega_{j}\right\}_{j \in Z}$ such that

$$
\left\langle g_{i}, \omega_{j}\right\rangle=\delta_{i . j} \quad \forall i, j \in Z,
$$

the system $\left\{\tilde{g}_{i}\right\}_{i \in Z}$ of the functionals is prolongation of the functional system, which is biorthogonal to the system $\left\{\widetilde{\omega}_{j}\right\}_{j_{\in}}$ such that

$$
\left\langle\tilde{g}_{i}, \widetilde{\omega}_{j}\right\rangle=\delta_{i . j} \quad \forall i, j \in Z,
$$

2) the functionals $\tilde{g}_{i}$ satisfy relations

$$
\left\langle\tilde{g}_{i}, u\right\rangle=\left\{\begin{array}{c}
\left\langle g_{i}, u\right\rangle \text { for } i \leq k \\
\left\langle g_{i+1}, u\right\rangle \text { for } i>k
\end{array} \quad \forall u \in S_{0}(\Xi) .\right.
$$

Proof. Using formula (37), we have

$$
\left\langle g_{i}, \omega_{j}\right\rangle=\omega_{j}\left(\xi_{i}\right)=\left\{\begin{array}{c}
1 \text { for } i=j \\
0 \text { for } i \neq j
\end{array}\right.
$$

Thus formula (43) is right. By formula (39) we obtain

$$
\left\langle\tilde{g}_{i}, \widetilde{\omega}_{j}\right\rangle=\widetilde{\omega}_{j}\left(\tilde{\xi}_{i}\right)=\left\{\begin{array}{l}
1 \text { for } i=j \\
0 \text { for } i \neq j
\end{array}\right.
$$

Now we see that relation (44) is fulfilled.

Taking into account relations (38) we deduce

$$
\begin{gathered}
\left\langle\tilde{g}_{i}, u\right\rangle=u\left(\tilde{\xi}_{i}\right)=u\left(\xi_{i}\right)=\left\langle g_{i}, u\right\rangle \text { for } i \leq k, \\
\left\langle\tilde{g}_{i}, u\right\rangle=u\left(\tilde{\xi}_{i}\right)=u\left(\xi_{i+1}\right)=\left\langle g_{i+1}, u\right\rangle \text { for } i>k .
\end{gathered}
$$

It follows that formula (45) is correct. This completes the proof.

\section{WAVELET DECOMPOSITION. FORMULAS OF RECONSTRUCTION}

Consider an operator $P$ for the projection of the space $S_{0}(\Xi)$ on the subspace $S_{0}(\tilde{\Xi})$,

$$
P u=\sum_{j \in \mathbb{Z}}\left\langle\tilde{g}_{j}, u\right\rangle \widetilde{\omega}_{j} \quad \forall u \in S_{0}(\Xi) .
$$

We also discuss the operator $Q=I-P$, where $I$ is the identical operator. The space $W_{0}=W_{0}(\Xi, \tilde{\Xi})=Q S_{0}(\Xi)$ is called the wavelet space of zero order. By (46) we have the direct decomposition

$$
S_{0}(\Xi)=S_{0}(\tilde{\Xi})+W_{0}(\Xi, \tilde{\Xi})
$$

that is the spline-wavelet decomposition of the space $S_{0}(\Xi)$. The space $S_{0}(\tilde{\Xi})$ is called the main space in decomposition (47). Consider the representation of element $u \in S_{0}(\Xi)$ on the basis $\left\{\omega_{i}\right\}_{i \in z}$ of the space $S_{0}(\Xi)$,

$$
u=\sum_{i \in \mathbb{Z}} c_{i} \omega_{i}, \quad c_{i}=\left\langle g_{i}, u\right\rangle .
$$

We suppose that the coefficients $a_{i}$ and $b_{i}$ in representations

$$
P u=\sum_{i \in \mathbb{Z}} a_{i} \widetilde{\omega}_{i}, \quad Q u=\sum_{i \in \mathbb{Z}} b_{i^{\prime}{ }^{\prime}} \omega_{i^{\prime} i^{\prime}}{ }^{\prime}
$$

where $a_{i}=\left\langle\tilde{g}_{j}, u\right\rangle, b_{i^{\prime} i^{\prime}}=\left\langle g_{i^{\prime} i^{\prime}}, Q u\right\rangle$ are known. According to formulas (42), (47) and (49) we have the representation

$$
\begin{gathered}
u=\sum_{i \in \mathbb{Z}} a_{i} \widetilde{\omega}_{i}+\sum_{i \in \mathbb{Z}} b_{i^{\prime} i^{\prime}} \omega_{i^{\prime} i^{\prime}}= \\
=\sum_{i^{\prime} \in \mathbb{Z} i^{\prime} \in \mathbb{Z}}\left(\sum_{i \in \mathbb{Z}} a_{i} p_{i, i^{\prime} i, i^{\prime}}+b_{i^{\prime} i^{\prime}}\right) \omega_{i^{\prime}{ }^{\prime}}\left(\sum_{i \in \mathbb{Z}} a_{i} p_{i, i^{\prime} i, i^{\prime}}\right.
\end{gathered}
$$

$\left.+b_{i^{\prime} i^{\prime}}\right) \omega_{i^{\prime} i^{\prime}}$.

By equating the right parts for relations (48) and (50) and taking into account the linear independence of the coordinate functions $\left\{\omega_{i}\right\}_{i \in Z}$ we have

$$
c_{j}=\sum_{i \in Z} a_{i} p_{i, j}+b_{j} \forall j \in \mathbb{Z} .
$$

Relations (51) are called reconstruction formulas.

Consider vectors $\boldsymbol{a}=\left(\ldots, a_{-2}, a_{-1}, a_{0}, a_{1}, a_{2}, \ldots\right), \boldsymbol{b}=$ $\left(\ldots, b_{-2}, b_{-1}, b_{0}, b_{1}, \ldots\right), \boldsymbol{c}=\left(\ldots, c_{-2}, c_{-1}, c_{0}, c_{1}, c_{2}, \ldots\right)$.

By formula (51) we have

$$
\boldsymbol{c}=\mathfrak{P}^{T} \boldsymbol{a}+\boldsymbol{b}
$$

Vector $\mathbf{c}$ is called the initial flow, the vectors $\mathbf{a}$ and $\mathbf{b}$ are named the main flow and wavelet flow respectively.

Lemma 8. In the spline-wavelet decomposition (47) of the space $S_{0}(\Xi)$ the reconstruction formulas have the form

$$
c_{j}=\left\{\begin{array}{c}
a_{j}+b_{j} \text { for } \leq k, \\
a_{j-1}+b_{j} \text { for } j \geq k+1 .
\end{array}\right.
$$

Proof. The usage of formulas (42), (52) gives relations (53).

\section{DECOMPOSITION FORMULAS}

Here we suppose that the coefficients $c_{k}$ in the decomposition of the element $u \in \mathrm{S}_{0}(\Xi)$ are known, $u=$ $\sum_{i} c_{i} \omega_{i}$. Taking into account the evident equalities $a_{i}=$ $\left\langle\tilde{g}_{j}, u\right\rangle$ and $u=\sum_{s \in \mathbb{Z}} c_{s} \omega_{s}$, we have

$$
a_{i}=\sum_{s \in \mathbb{Z}} c_{s}\left\langle\tilde{g}_{i}, \widetilde{\omega}_{s}\right\rangle \text {. }
$$


By formula (51) we deduce $b_{j}=c_{j}-\sum_{i} p_{i, j} \sum_{s} c_{s}\left\langle\widetilde{g}_{i}, \widetilde{\omega}_{s}\right\rangle$. Using (54) and applying the notation $q_{i, s}=\left\langle\tilde{g}_{i}, \omega_{s}\right\rangle$, we have the decomposition formulas

$a_{i}=\sum_{s \in \mathbb{Z}} c_{s} q_{i, s}, b_{j}=c_{j}-\sum_{s} c_{s} \sum_{s} p_{i, j} q_{i, s}$.

By a matrix $\mathfrak{Q}=\left(q_{i, s}\right), \mathfrak{Q}=\left(q_{i, s}\right)$, we rewrite formulas (55) in the form

$$
\boldsymbol{a}=\mathfrak{Q} \boldsymbol{c}, \quad \boldsymbol{b}=\boldsymbol{c}-\mathfrak{P}^{T} \mathfrak{Q} \boldsymbol{c} .
$$

Theorem 7. The next formulas are right:

$$
\begin{gathered}
q_{i, i}=1 \text { for } i \leq k, \quad q_{i, i+1}=1 \text { for } i>k, \\
q_{i, s}=0 \text { in other cases. }
\end{gathered}
$$

Proof. By Theorem 4 and formulas (40) - (41), we have

$$
\left\langle\tilde{g}_{i}, \omega_{s}\right\rangle=\delta_{i, s} \text { for } i \leq k,\left\langle\tilde{g}_{i}, \omega_{s}\right\rangle=\delta_{i+1, s} \text { for } i>k \text {. }
$$

Relation (59) is equivalent to formulas $(57)-(58)$. This completes the proof.

Let $A$ be a matrix with elements $a_{i j}$. By definition put $[A]_{i j}=$ $a_{i j}$. By (40)-(42) we have $\quad\left[\mathfrak{P}^{T} \mathfrak{Q}\right]_{i j}=\sum_{s_{\leq k-1}} \delta_{s, i} q_{s j}+$ $\left(\delta_{k, i}+\delta_{k, i-1}\right) q_{k j}+\sum_{s_{\geq k+1}} \delta_{s, i-1} q_{s j}$. Taking into account formulas $(57)-(58)$ we obtain $\left[\mathfrak{P}^{T} \mathfrak{Q}\right]_{i j}=$

$$
\left\{\begin{array}{c}
\delta_{i, j} \text { for } i \leq k \\
\delta_{k, j} \text { for } i=k+1 \\
\delta_{i, j} \text { for }>k+1
\end{array}\right.
$$

for all $j \in \mathrm{Z}$. By (60) we have

$$
\begin{gathered}
{\left[I-\mathfrak{P}^{T} \mathfrak{Q}\right]_{k+1, k}=-1,\left[I-\mathfrak{P}^{T} \mathfrak{Q}\right]_{k+1, k+1}=+1 .(61)} \\
{\left[I-\mathfrak{P}^{T} \mathfrak{Q}\right]_{i, j}=0 \text { for }(i, j) \in\left\{\left(i^{\prime}, j^{\prime} i^{\prime}, j^{\prime}\right) \mid i^{\prime}, j^{\prime} \in Z\right\}} \\
\backslash\{(k, k+1),(k+1, k+1)\} .
\end{gathered}
$$

Theorem 8. The decomposition formulas for the spline wavelet decomposition (56) have the form

$$
\begin{gathered}
a_{i}=\left\{\begin{array}{l}
c_{i} \quad \text { for } i \leq k, \\
c_{i+1} \text { for } j \geq k+1
\end{array}\right. \\
b_{j}=0 \text { for } j \neq k+1, \quad b_{k+1}=c_{k_{+1}}-c_{k} .
\end{gathered}
$$

Proof. By representations (56) and (60) - (62) we simply deduce relations $(63)-(64)$.

\section{Spline-Wavelet Decomposition of Finite Flow}

For a natural number $m$ by definition put $J_{m}=\{0,1, \ldots, m\}$. Let $M$ be the natural, $M \geq 3$. We denote $a=\xi_{0}, b=\xi_{M+1}$. Consider set $\llbracket a, b \rrbracket=\left\{\xi_{i}\right\}_{i} \in J_{M_{+1}}$. The set $\llbracket a, b \rrbracket$ is named a grid segment. Consider the coordinate splines $\left\{\omega_{j}(t)\right\}_{j} \in$ $J_{M_{+1}}$. The linear hull of these splines is denoted $C \llbracket a, b \rrbracket$. Using the evident relations $\omega_{j}\left(\xi_{s}\right)=\delta_{s, j}, s \in J_{M+1}$, we discuss the linear functionals defined by the formula $\left\langle g^{(i)}, u\right\rangle=u\left(\xi_{i}\right) \quad \forall u \in C \llbracket a, b \rrbracket$.

We have relations $\left\langle g^{(i)}, \omega_{j}\right\rangle=\delta_{i, j} \forall i, j \in J_{M_{+1}}$. Let $k$ be a natural number satisfying the condition $0 \leq k \leq M-1$. As before we delete the knot $\eta=\xi_{k+1}$ from the grid $\llbracket a, b \rrbracket$. As a result we obtain the grid $\hat{\xi}_{j}=\xi_{j}$ for $0 \leq j \leq k$, and $\hat{\xi}_{j}=$ $\xi_{j+1}$ fork $+1 \leq j \leq M+1$, and $\hat{\Xi}: a=\xi_{0}<\xi_{1}<\ldots<$ $\xi_{M}=b$. Consider functions $\hat{\omega}_{j}(t)$ defined by the relations

$$
\begin{array}{lll}
\widehat{\omega}_{j}(t)=1 & \text { for } & \left.t \in \hat{\xi}_{j}, \hat{\xi}_{j+1}\right), \\
\widehat{\omega}_{j}(t)=0 & \text { for } & \left.t \notin \hat{\xi}_{j}, \hat{\xi}_{j+1}\right),
\end{array}
$$

where $0 \leq j \leq M$. For all $t \in\left[\xi_{0}, \xi_{M+2}\right)$ we have

$$
\widehat{\omega}_{k}(t)=\omega_{k}(t)+\omega_{k+1}(t)
$$

By (66) we obtain

$$
\begin{aligned}
& \widehat{\omega}_{j}(t)=\omega_{j}(t) \text { for } \quad 0 \leq j \leq k-1, \\
& \widehat{\omega}_{j}(t)=\omega_{j+1}(t) \text { for } \quad k+1 \leq j \leq M,
\end{aligned}
$$

where $t \in\left[\xi_{0}, \xi_{M+2}\right)$. Formulas $(67)-(68)$ are calibration relations. Now we see that in the case of a finite grid the discussion repeats the arguments of the case of the infinite grid. Therefore we would like to formulate the final results of our discussion. Let $\overline{\mathbf{c}}$ be an initial flow, $\hat{\mathbf{a}}$ be a main flow and $\overline{\mathbf{b}}$ be a wavelet flow, where $\widehat{\boldsymbol{a}}=\left(\hat{a}_{0}, \hat{a}_{1}, \hat{a}_{2}, \ldots, \hat{a}_{M}\right), \overline{\boldsymbol{b}}=$ $\left(\bar{b}_{0}, \bar{b}_{1}, \bar{b}_{2}, \ldots, \bar{b}_{M}, \bar{b}_{M+1}\right)$,

$$
\overline{\boldsymbol{c}}=\left(\bar{c}_{0}, \bar{c}_{1}, \bar{c}_{2}, \ldots, \bar{c}_{M}, \bar{c}_{M+1}\right) .
$$

Consider the rectangular matrix $\hat{P}$ of the size $(M+1) \times$ $(M+2)$, which has a form $\widehat{\mathfrak{P}}=\left(\hat{p}_{i, j}\right)_{i \in J_{M}, j \in J_{M+1}}$,where $\hat{p}_{i, j}=1$ for $i \in J_{k}, \quad \hat{p}_{i-1, i}=1$ for $i \in J_{M+1} \bigvee_{k}$,

$$
\begin{gathered}
\hat{p}_{i, j}=0 \text { for }(i, j) \in\left\{\left(i^{\prime}, j^{\prime}\right) \mid i^{\prime} \in J_{M}, j^{\prime} \in J_{M+1}\right\} \\
\backslash\left(\left\{\left(i^{\prime}, j^{\prime}\right) \mid i^{\prime}=j^{\prime}, j^{\prime} \in J_{k}\right\} \cup\right.
\end{gathered}
$$$$
\left.\cup\left\{\left(i^{\prime}, j^{\prime}\right) \mid i^{\prime}=j^{\prime}-1, j^{\prime} \in J_{M_{+1}} V_{k}\right\}\right) \text {. }
$$

Let $\overline{\mathfrak{Q}}$ also be a rectangular matrix of size $(M+1) \times(M+2)$

such that $\overline{\mathfrak{Q}}=\left(\bar{q}_{i, j}\right)_{i \in J_{M}, j \in J_{M+1}}$, where

$$
\begin{array}{r}
\bar{q}_{i, i}=1 \text { for } i \in J_{k}, \bar{q}_{i, i+1}=1 \text { for } i \in J_{M} \bigvee_{k}, \\
\bar{q}_{i, j}=0 \text { for }(i, j) \in\left\{\left(i^{\prime}, j^{\prime}\right) \mid i^{\prime} \in J_{M}, j^{\prime} \in J_{M+1}\right\} \\
\backslash\left(\left\{\left(i^{\prime}, j^{\prime}\right) \mid i^{\prime}=j^{\prime}, j^{\prime} \in J_{k}\right\} \cup\right. \\
\left.\cup\left\{\left(i^{\prime}, j^{\prime}\right) \mid j^{\prime}=i^{\prime}+1, i^{\prime} \in J_{M} \bigvee_{k}\right\}\right) .
\end{array}
$$

In the discussed case we obtain the decomposition formulas

$$
\widehat{\boldsymbol{a}}=\overline{\mathfrak{Q}} \overline{\mathbf{c}}, \quad \overline{\boldsymbol{b}}=\overline{\mathbf{c}}-\widehat{\mathfrak{P}}^{T} \overline{\mathfrak{Q}} \overline{\overline{\mathbf{c}}},
$$


and the reconstruction formulas

$$
\overline{\overline{\mathbf{c}}}=\widehat{\mathfrak{P}}^{T} \widehat{\boldsymbol{a}}+\overline{\boldsymbol{b}} .
$$

It is clear to see that $\hat{\mathbf{P}}^{T} \overline{\mathbf{Q}}$ is the square matrix of the size $(M+2) \times(M+2)$. By $(69)-(72)$ we have $\left[\widehat{\mathfrak{P}}^{T} \overline{\mathbf{Q}}\right]_{i, i}=$ 1 for $i \in J_{M+1} \backslash\{k+1\}$ and

$$
\left[\widehat{\mathfrak{P}}^{T} \overline{\mathfrak{Q}}\right]_{k+1, k}=1 .
$$

Other values $\left[\widehat{\mathfrak{P}}^{T} \overline{\mathfrak{Q}}\right]_{i j}$ equal zero, i.e. $\left[\widehat{\mathfrak{P}}^{T} \overline{\mathfrak{Q}}\right]_{i j}=$ $0 \quad f o r(i, j) \in\left\{i^{\prime}, j^{\prime} \in J_{M_{+1}}\right\}$

$$
\backslash\left\{i^{\prime}=j^{\prime}, j^{\prime} \in J_{M_{+1}}\right\} \cup\{(k+1, k)\} .
$$

By (75) - (76) we obtain

$$
\begin{aligned}
{\left[I-\widehat{\mathfrak{P}}^{T} \overline{\mathfrak{Q}}\right]_{k+1, k} } & =-1,\left[I-\widehat{\mathfrak{P}}^{T} \overline{\mathfrak{Q}}\right]_{k+1, k+1}=+1,(77 \\
{\left[I-\widehat{\mathfrak{P}}^{T} \overline{\mathfrak{Q}}\right]_{k+1, k}=} & 0 \text { for }(i, j) \\
& \in\left\{i^{\prime}, j^{\prime} \in J_{M+1}\right\} \\
& \backslash\{(k+1, k),(k+1, k+1)\} .
\end{aligned}
$$

Theorem 9. If $0 \leq k \leq M-1$ then 1) the decomposition formulas can be written in the form

$$
\begin{aligned}
& \hat{a}_{i}=\bar{c}_{i} \text { for } i \in J_{k}, \hat{a}_{i}=\bar{c}_{i+1} \text { for } i \in J_{M} \bigvee_{k}, \\
& \bar{b}_{i}=0 \text { for } i \in J_{M+1} \backslash\{k+1\}, \bar{b}_{k+1}=\bar{c}_{k+1}-\bar{c}_{k},
\end{aligned}
$$

2) the reconstruction formulas can be represented in form

$$
\overline{\boldsymbol{c}}_{\boldsymbol{i}}=\left\{\begin{array}{l}
a_{i}+b_{i} \text { for } \quad i \in J_{k} \\
a_{i-1}+b_{i} \text { for } i \in J_{M+1} \bigvee_{k} .
\end{array}\right.
$$

Proof. According to formulas (69) - (70), the rectangular matrix $\mathrm{P}$ has units in the places $(i, i)$ for $i=0,1, \ldots, k$, and also in the places $(i, i+1)$ for $i=k, k+1, \ldots, M$.The remaining elements are zero. According to formulas (71) - (72), the matrix $Q$ has units in places $(i, i)$ for $i=0,1, \ldots, k$, and also in places $(i, i+1)$ for $i=k+1, \ldots, M$.The remaining elements are zero. After transposing the matrix $P$ and multiplying it by the matrix $Q$, we obtain a matrix that has units in places $(i, i)$ for $i=0,1, \ldots, k, k+2, \ldots, M+1$. In addition, there is a unit in the place $(k+1, k)$. The remaining elements are equal to zero (see formulas (75) - (76)). Due to this, subtraction of this product from the identity matrix leads to a matrix with only two nonzero elements. In the place of $(k+1, k)$ it is the value -1 , and in place of $(k+1, k+1)$ it is placed +1 (see formulas (77)). These considerations lead to relations (79) $-(81)$. This completes the proof.

\section{ILLUSTRATIVE EXAMPLE}

In the illustrative example below, we used the following data: $m=6, k=3, \Xi=\left\{\xi_{i}\right\}_{i=0,1, \ldots, 7}, \xi_{i}=0.2 i, c_{i}=\sin \left(\xi_{i}\right)$, $i=0,1, \ldots, 7$.

The results are presented in Table No. 1. The first column contains the numbers of the components of the flows (vectors) appearing in the remaining columns. The components of the initial flow $\overline{\mathbf{c}}$ is shown in the second column of the table. The main flow components fill the third column. The components of the wavelet flow $\overline{\mathbf{b}}$ are done in the fourth column. This ends the decomposition. The fifth column contains the result of the reconstruction. This result is given as a flow $\overline{\mathbf{c c}}$.

Table 1. Computational results.

\begin{tabular}{|c|c|c|c|c|}
\hline$i$ & $\bar{c}_{i}$ & $\hat{a}_{i}=[\bar{Q} \bar{c}]_{i}$ & $\begin{array}{c}\bar{b}_{i}= \\
=\left[I-\hat{P}^{T} \bar{Q} \bar{c}\right]_{i}\end{array}$ & $\begin{array}{l}{[\overline{c c}]_{i}=} \\
=\left[\hat{P}^{T} \hat{a}\right]_{i}+\bar{b}_{i}\end{array}$ \\
\hline 0 & 0.0000 & 0.0000 & 0.0000 & 0.0000 \\
1 & 0.1987 & 0.1987 & 0.0000 & 0.1987 \\
2 & 0.3894 & 0.3894 & 0.0000 & 0.3894 \\
3 & 0.5646 & 0.5646 & 0.0000 & 0.5646 \\
4 & 0.7173 & 0.8415 & 0.1527 & 0.7173 \\
5 & 0.8415 & 0.9320 & 0.0000 & 0.8415 \\
6 & 0.9320 & 0.9854 & 0.0000 & 0.9320 \\
7 & 0.9854 & & 0.0000 & 0.9854 \\
\hline
\end{tabular}

\section{Remarks to Table 1.}

1. The wavelet flow, together with the main flow, allows the receiver to reconstruct the initial flow exactly. This is the value of the wavelet decomposition. This property is clearly seen in Table 1 . In it, the fifth column completely coincides with the second, which is consistent with the theoretical results obtained in this work.

2. The program is written in the Maple-17 system (see [40]). The calculation were carried out on an HP 27p251ur monoblock, Digits $=10$.

\section{THE NUMBER OF OPERATIONS}

First, we consider the adaptive grid construction algorithms described in the proof of Lemma 1.

Theorem 10. If the conditions of Theorem 3 are fulfilled then for $f=u^{\prime}$ hold the next relations

$$
\begin{gathered}
N(f)=N_{\text {add }}(f)=N_{\text {mul }}(f)=K_{u}^{\prime}(\eta)+M+2, \\
N_{\text {comp }}(f)=2 N_{\text {mul }}(f) .
\end{gathered}
$$

The proof of formulas (82) follows from Lemma 4 and Theorem 3. 
Note that in order to apply this theorem it is required to know the corresponding derivative functions (at least approximately). Now we discuss another approach.

Consider the fine equidistant grid segment $\llbracket a, b \rrbracket=$ $\left\{a=\xi_{0}, \xi_{1}, \ldots, \xi_{M+1}=b\right\}$, where $\xi_{i}=i \square, \square=(b-a) /$ $(M+1)$. It is easy to see that the proof of Lemma 1 can be carried out so that the grid $\tilde{X}$ is a subset of the set $\Xi$. In this case, the proof of Lemma 1 can be considered as an algorithm for constructing the grid $\tilde{X}$. The number of operations in this algorithm is of the order of $M$. This algorithm can be applied to approximate the function $u$ in the same way as was done in the previous theorems. But for this you need to know the derivatives of the function $u$. Now we consider the construction of the approximation of this function in a situation in which the mentioned derivatives are not known. We discuss the function

$$
\begin{aligned}
& \Phi\left(\xi, x_{0}, x_{1}\right)=u(\xi)-u\left(x_{0}\right)-\frac{u\left(x_{1}\right)-u\left(x_{0}\right)}{x_{1}-x_{0}}\left(\xi-x_{0}\right) \\
& \forall \xi \in\left[x_{0}, x_{1}\right], a \leq x_{0}<x_{1} \leq b, x_{0}, x_{1}, \xi \in \Xi
\end{aligned}
$$

The value $\Phi\left(\xi, x_{0}, x_{1}\right)$ will be called the deviation of the chord $L\left(x_{0}, x_{1}\right)$ from the function $u$ at the point $\xi$. Consider the process of constructing the grid $\tilde{X}=\tilde{X}(u, \varepsilon)$, which consists of the fact that after finding the knot $\tilde{x}_{s}$ knot $\tilde{x}_{s+1}$ is searched for by using the two-point criterion:

1) $\left|\Phi\left(\xi, \tilde{x}_{s}, \tilde{x}_{s+1}\right)\right| \leq \varepsilon$ for $\tilde{x}_{s}<\xi<x$,

2) a value $\delta>0$ exists such that $\left|\Phi\left(\xi, \tilde{x}_{s}, \tilde{x}_{s+1}\right)\right| \geq \varepsilon$ for $\tilde{x}_{s+1}<\xi<\tilde{x}_{s+1}+\delta$.

This criterion is checked on the initial grid $\Xi$. We have $\tilde{X}(u, \varepsilon) \subset \Xi$. Consider the operation of searching for the difference

$\Phi\left(\xi_{i}, \tilde{x}_{s}, \xi_{j}\right)=u\left(\xi_{i}\right)-u\left(\tilde{x}_{s}\right)-\frac{u\left(\xi_{j}\right)-u\left(\tilde{x}_{s}\right)}{\xi_{j}-\tilde{x}_{s}}\left(\xi_{i}-\tilde{x}_{s}\right)$

The considered algorithm is iterative in nature and consists of the sequential selection of suitable knots of the source grid. This algorithm will be described in more detail.

Algorithm $(P)$ :

0 . Let $\tilde{x}_{0}:=a$.

1. Suppose the knots $\tilde{x}_{0}<\tilde{x}_{1}<\ldots<\tilde{x}_{s}$ of the desired adaptive grid has already been found, and $\tilde{x}_{s}=\xi_{j_{s}}$. If $j_{s}+1 \leq$ $M$, then we change the parameter $j$ in formula (84), sequentially taking $j=j_{s}+2, j_{s}+3, \ldots, M+1$, and checking every time the fulfillment of all inequalities

$$
\left|\Phi\left(\xi_{i}, \tilde{x}_{s}, \xi_{j}\right)\right| \leq \varepsilon, \quad i=j_{s}+1, j_{s}+2, \ldots, j-1 .
$$

If all inequalities (85) are satisfied, and $j<M+1$, then we add a unit to $j$ and go back to checking inequalities (85), i.e. repeat point 1 .

2. This process is interrupted in one of two cases:

a/. It turns out that $j=M+1$. In that case, select the knot $\tilde{x}_{s+1}=\xi_{M+1}$ and put $K=s$. The adaptive griding process $\tilde{X}$ is finished.

$\mathrm{b} /$. For $j<M+1$, at least one of the inequalities (85) is violated. In this case, we select the knot $\tilde{x}_{s+1}=\xi_{j-1}$. We reassign variables by setting $s:=s-1, j_{s}:=j-1$ and go to step 1, i.e. make the next iteration cycle.

It is clear to see that the previous discussion proves the next assertion.

Lemma 9. Under implementing algorithm (P)

a number $\mathrm{V}$ of calculations (84) is determined by the formula

$$
2 \mathrm{~V}=\sum_{s=0}^{K}\left(j_{s+1}-j_{S}\right)^{2}-(M+1) .
$$

Proof. By assumption, the grid $\Xi$ is equidistant grid, and $\tilde{X}$ is a subset of the set $\Xi$; therefore the expression $\left(\tilde{x}_{s+1}\right.$ $\left.-\tilde{x}_{s}\right) / h$ is an integer number. Without loss of generality, we assume that $\tilde{x}_{s}=j_{s} \square$. It is obvious that $\sum_{s=0}^{K}\left(j_{s+1}-j_{s}\right) h=$ $\sum_{s=0}^{K}\left(\tilde{x}_{s+1}-\tilde{x}_{s}\right)=\tilde{x}_{K+1}-\tilde{x}_{0}=b-a$. Thus,

$\sum_{s=0}^{K}\left(j_{s+1}-j_{s}\right)=(b-a) / \square=M+1$.

Between the knots $\tilde{x}_{s}$ and $\tilde{x}_{s+1}$ there is $\left(\tilde{x}_{s+1}-\tilde{x}_{s}\right) /$ $h-1=j_{s+1}-j_{s}-1$ knots of equidistant grid. In the process of the building of the next knot for the adaptive grid we draw chords $L_{j_{s}, i}$ through the knots $\xi_{j_{s}}=\tilde{x}_{s}$ and $\xi_{i}$ for $i \in\left\{j_{s}+\right.$ $\left.2, j_{s}+3, \ldots, j_{s+1}\right\}$. For each chord $L_{j_{s}, i}$ we calculate expression (84) $i-j_{s}-1$ times. Thus, the number of $V_{s}$ calculations of expression (84) to get the next knot $\tilde{x}_{s+1}$ is defined by the formula $V_{s}=\sum_{i=j_{s}+2}^{j_{S+1}}\left(i-j_{s}-\right.$ 1) $=\sum_{i^{\prime}=1}^{j_{s+1}-j_{s}-1} i^{\prime}=\left(j_{s+1}-j_{s}-1\right)\left(j_{s+1}-j_{s}\right) / 2$.

To obtain the number of $\mathrm{V}$ calculations of expression (84) for building the entire grid $\tilde{X}$ it remains to calculate the sum $V=\sum_{s=0}^{K} V_{s}$. Given relation (87), we derive formula (86). This completes the proof.

Let us estimate lower bound and upper bound of expression (86).

For this we need the following statement.

Lemma 10. Let $\boldsymbol{y}=\left(y_{0}, y_{1}, \ldots, y_{n}\right)$ be $n+1$ dimensional vector, and let $C$ be a constant, $C>n+1$.The quadratic form

discussed on the set

$$
Q_{n}(y)=\sum_{s=0}^{n} y_{s}^{2}
$$

$$
\Omega_{n, C}=\left\{y \mid y_{s} \geq 1, \sum_{s=0}^{n} y_{s}=C\right\}
$$

satisfies to the inequalities

$$
C^{2} /(n+1) \leq\left. Q_{n}(y)\right|_{y \in \Omega_{n, C}} \leq(C-n)^{2}+n
$$

The inequality on the left of (90) turns into the equality in the point $y^{*}=\left(y_{0}^{*}, y_{1}^{*}, \ldots, y_{n}^{*}\right) \quad$ with identical components $y_{s}^{*}=C /(n+1) \forall s \in\{0,1,2, \ldots, n\}$.

The inequality on the right part of (90) turns into equality in the points $(C-n) e^{(s)}+e$, where $e=(1,1, \ldots, 1), e^{(s)}=$ $\left(e_{j}^{(s)}\right)_{j \in\{0,1,2, \ldots, n\}}$ are $n+1$-dimensional vectors, and $e_{j}^{(s)}=\delta_{s, j}$ is the Kronecker symbol.

Proof. Consider the conditional extremum problem for functions (88) under the condition

$$
\sum_{s=0}^{n} y_{s}=C \text {. }
$$

To find a critical point we introduce the function $\Psi(y, \lambda)=Q_{n}(y)+\lambda\left(\sum_{s=0}^{n} y_{s}-C\right)$ and equate its derivatives to zero. As a result, we get equivalence $\frac{\partial \Psi}{\partial y_{s}}=0 \quad<=>y_{s}^{* *}=-\lambda / 2 \quad \forall s \in\{0,1,2, \ldots, n\}$. 
into condition (91), we find $\lambda / 2=-C /(n+1)$. . From (92) we find $y_{s}^{* *}=C /(n+1)>1 \forall s \in\{0,1,2, \ldots, n\}$.

So, the critical point is $y^{* *}=\left(y_{0}^{* *}, y_{1}^{* *}, \ldots, y_{n}^{* *}\right)$, it is a single and an interior point of the set (89). Clearly, it is a conditional minimum point. We have $Q_{n}\left(y^{* *}\right)=C^{2} /(n+$ 1) $\leq\left. Q_{n}(y)\right|_{y \in \Omega_{n, C}}$.

The left inequality in formula (90) is proved.

The proof of the right-hand side is obtained by decreasing the induction on dimension $n$. From the previous one it is clear that the greatest value must lie on the boundary of the region $\Omega_{n, C}$. Consider, for example, the part of the boundary defined by the equality $y_{n}=1$. This leads to a problem similar to the previous one, but on a unit of a smaller dimension, it is required to find a minimum of a function

$\sum_{s=0}^{n} y_{s}^{2}+1$ on the set $\Omega_{n-1, C-1}$ of the form $\left\{\left(y_{0}, y_{1}, \ldots, y_{n-1}\right) \mid \sum_{s=0}^{n-1} y_{s}=C-1, y_{j} \geq 1, j=\right.$ $, 1,2, \ldots, n-1\}$.

Similarly to previous reasoning in this case, we conclude that the greatest value should be reached at the border. Continuing to downgrade we eventually arrive at the function $Q_{n}\left(y_{0}, 1,1, \ldots, 1\right)=Q_{0}\left(y_{0}\right)+n=y_{0}^{2}+n$ under the condition $y_{0}=C-n$, so the highest value is reached at the point $(C-n, 0,0, \ldots, 0)$ and is equal to $(C-n)^{2}+n$. Similar reasoning for other parts of the border, they produce the same result. This concludes the proof.

Theorem 11. Number $V$ of calculations of expression (84) satisfies the inequalities

$$
\frac{M+1}{K+1}(M-K) \leq 2 V \leq(M-K+1)(M-K)
$$

and the left side of this inequality turns into equality, if found in accordance with the algorithm $(P)$ the adaptive grid turns out to be uniform. The right inequality (93) turns into equality if the mentioned grid can be formed removing one consecutive group of knots from the source grid.

Proof. To estimate the value of $V$, we use Lemma 10

Setting $y_{s}=j_{s+1}-j_{s}$, according to relation (87), we have $\sum_{s=0}^{K} y_{s}=\sum_{s=0}^{K}\left(j_{s+1}-j_{s}\right)=M+1$.

Since the various knots of the initial grid do not coincide, the inequalities

$$
y_{s} \geq 1 \forall s \in\{0,1,2, \ldots, K\}
$$

are right.

Obviously, by relations (94) and (95) for $n=K, C=M+$ 1 the expression $Q_{K}(y)=\sum_{s=0}^{K} y_{s}^{2} \quad$ satisfies the conditions of Lemma 10, so that the inequalities

$(M+1)^{2} /(K+1) \leq Q_{K}(y) \leq(M-K+1)^{2}+K$

are right. By the representation of $2 V=Q_{K}(y)-(M+1)$ we arrive at the inequalities (93). According to

Lemma 10, the lower bound for $V$ is reached when all numbers $y_{s}$ are the same, i.e. when the adaptive grid $\widetilde{X}$ turns out to be uniform.

Upper bound in this evaluation is achieved when all components of the vector $\boldsymbol{y}=\left(y_{0}, y_{1}, \ldots, y_{K}\right)$ are equal to unity, except for one, which is equal to $C-n=M-K+1$. The number $i$ of this component may be any number from the set $\{0,1,2, \ldots, K\}$. For example, for the $\mathrm{i}$-th components have $j_{i+1}-j_{i}=M-K+1$, i.e. between the knots $\tilde{x}_{i}$ and $\tilde{x}_{i+1}$ are deleted $M-K$ knots of the initial grid. Between the other pairs of neighboring knots, adaptive grid $\tilde{X}$ knots were not deleted. This completes the proof.

\section{CONVEX FLOWS}

Let $\varepsilon$ be a positive value. Discuss the function $u(x)$ on the set $\Xi$.

Definition 1. We say that the function $u(x)$ is weakly convex (up) on the set $\Xi$ iffor any $a^{\prime}, b^{\prime} \in \Xi, a^{\prime}<b^{\prime}$, inequality

$$
u(\xi) \geq u\left(a^{\prime}\right)+\frac{u\left(b^{\prime}\right)-u\left(a^{\prime}\right)}{b^{\prime}-a^{\prime}}\left(\xi-a^{\prime}\right)
$$

$\forall \in$ ?,$a^{\prime}<\xi<b^{\prime}$

is right.

In this paragraph, we assume that the arguments of the considered functions lie in the set $\Xi$. In particular, referring to representation (83), we assume that $x_{0} \leq \xi \leq x_{1}, x_{0}<$ $x_{1}, x_{0}, \xi, x_{1} \in \Xi$.

It follows from relation (83) that the relations $\Phi\left(x_{0}, x_{0}, x_{1}\right)=\Phi\left(x_{1}, x_{0}, x_{1}\right)=0$ are valid. If the function $u(x)$ is weakly convex (up), then

$$
\Phi\left(\xi, x_{0}, x_{1}\right) \geq 0 \quad \text { for } \quad \xi \in\left[x_{0}, x_{1}\right] .
$$

To prove relation (88), it is sufficient to use the definition of the weak convexity (87), setting $a^{\prime}=x_{0}, b^{\prime}=x_{1}$.

Consider the supremum $F(x)$ of the function $\Phi(\xi, a, x)$ with respect to $\xi \in(a, x) \cap \Xi$, where $a<x$,

$$
F(x)=\sup _{\xi \in(a, x) \cap \Xi} \Phi(\xi, a, x) .
$$

Theorem 12. If the function $u(x)$ is weakly convex (up) then $F\left(x^{\prime}\right) \leq F\left(x^{\prime \prime}\right) \forall x^{\prime}, x^{\prime \prime} \in(a, b) \cap \Xi, x^{\prime}<x^{\prime \prime}$.

Proof. Using the weak convexity of the function $u(x)$, we implement inequality (96) for $a^{\prime}=a, b^{\prime}=x$. As a result we have

$$
u(\xi) \geq u(a)+\frac{u(x)-u(a)}{x-a}(\xi-a) \forall \xi \in(a, x) \cap \Xi .
$$

Taking into account that $\xi-a$ is a positive value, by (99) we have

$$
\frac{u(\xi)-u(a)}{\xi-a} \geq \frac{u(x)-u(a)}{x-a} \quad \forall \xi \in(a, x) \cap \Xi .
$$

Let $\xi=x^{\prime}, x=x^{\prime \prime}$. As a result of (100) we deduce

$$
\frac{u(x)-u(a)}{x^{\prime}-a} \geq \frac{u\left(x^{\prime \prime}\right)-u(a)}{x^{\prime \prime}-a} \quad \forall x^{\prime} \in\left(a, x^{\prime \prime}\right) \cap \Xi
$$

Multiplying inequality (101) by $-(\xi-a)$ for $\xi \in$ $\left(a, x^{\prime \prime}\right)$ and adding to both parts the value $u(\xi)-u(a)$, we obtain the relation

$$
\begin{aligned}
& u(\xi)-u(a)-\frac{u(x)-u(a)}{x^{\prime}-a}(\xi-a) \leq u(\xi)-u(a)- \\
& \frac{u\left(x^{\prime \prime}\right)-u(a)}{x^{\prime \prime}-a}(\xi-a) \forall x^{\prime}, \xi \in\left(a, x^{\prime \prime}\right) \cap \Xi . \\
& \text { Relation }(102) \text { can be written in the form } \\
& \Phi(\xi, a, x) \leq \Phi\left(\xi, a, x^{\prime \prime}\right) \forall x^{\prime}, \xi \in\left(a, x^{\prime \prime}\right) \cap \Xi . \\
& \text { By definition }(98) \text { we have } \\
& \Phi\left(\xi, a, x^{\prime}\right) \leq F\left(x^{\prime \prime}\right) \forall \xi \in\left(a, x^{\prime}\right) \cap \Xi . \\
& \text { Using relation }(103), \text { we deduce } \\
& \Phi\left(\xi, a, x^{\prime}\right) \leq F\left(x^{\prime \prime}\right) \forall x^{\prime}, \xi \in\left(a, x^{\prime \prime}\right) \cap \Xi .
\end{aligned}
$$


Passing to the exact upper bound of the left side of inequality (105) according to $\xi \in\left(a, x^{\prime}\right) \cap \Xi$, we obtain relation $F\left(x^{\prime}\right) \leq F\left(x^{\prime \prime}\right) \forall x^{\prime} \in\left(a, x^{\prime \prime}\right) \cap \Xi$.

This completes the proof.

Consider the algorithm for finding an adaptive grid, based on the idea of bisection. The algorithm described here is suitable for the weakly convex function. It is significantly more economical than the previous one.

We suppose that $b=\xi_{j_{0}}, F\left(\xi_{j_{0}}\right) \geq \varepsilon$.

\section{Algorithm $(Q)$ :}

0 . We accomplish assignments $x_{0}=\xi_{0}, s:=0$.

1. Suppose that the previous $\operatorname{knot} \tilde{x}_{s}=\xi_{i}$ has been found. Thus, $F\left(\xi_{i}\right) \leq \varepsilon$.

2. We do assignment $j:=j_{0}$.

3. We calculate $k=[(i+j) / 2]$. If $F\left(\xi_{k}\right) \leq \varepsilon$ then we put $i$ $:=k$, but if $F\left(\xi_{k}\right)>\varepsilon$ then we put $j:=k$. If $j-i>1$ then we go to point 3 , but if $j=i+1$ then we put $\tilde{x}_{s+1}=\xi_{i}$.

Thus the next knot of the adaptive grid has been found.

4. If $i<j_{0}-1$ then we put $s:=s+1$ and go to point 2, but if $i$ $=j_{0}-1$ then the Algorithm $(Q)$ has finished, the adaptive grid has been obtained. A next fragment of initial grid will begin with $\operatorname{knot} a:=b$.

It is easy to see that the next assertion can be proved.

Theorem 13. Suppose the algorithm $(Q)$ is implemented to a weakly convex flow $\left\{u\left(\xi_{i}\right)\right\}, i \in\{0,1, \ldots, M\}$, where $M=2^{k}$. Then the number $\hat{V}$ of calculations of formula (48)satisfies to inequality

$$
2^{k^{+1}}-k-2 \leq \widehat{V} \leq k\left(2^{k}-1\right)
$$

Inequality (89) is exact: the left and right parts can be reached for some flows.

By formula (106) we have the next assertion.

Corollary 1. In the general case (that is, when $M$ is not necessarily a power of two) under the conditions of Theorem 13 , the estimate $2(M-1)-\left\lceil\log _{2} M\right\rceil \leq \hat{V} \leq(M-$ 1) $\left\lceil\log _{2} M\right\rceil$ is right.

\section{CONCLUSION}

This paper is proposed an adaptive wavelet decomposition using an adaptive compression algorithm for a flow of numerical information of length $\mathrm{M}$ with complexity $O(M)$ and with a given precision of $\varepsilon>0$. The natural source of optimization is the adaptive processing for the initial data flow. It is clear to see that the processing speed of the decomposition and length $\mathrm{K}$ of the compression result are very important. It is also important the possibility of restoring the initial flow with a given precision of $\varepsilon>0$ from the obtained compressed flow. On other hand, the wavelet flow, together with the main flow, allows the receiver to reconstruct the initial flow exactly (the remarkable property of the wavelet expansion). However, the wavelet flow is too long, it can only be transmitted by a special request from the receiver.
This remarkable property of the wavelet expansion brings us to the question of the complexity of the algorithm for the expansion. The classical wavelet decomposition removes the odd components of the wavelet flow. This decomposition is not adaptive because it does not take into account the properties of the initial flow. With a non-classical approach to adaptive wavelet expansion is possible (see V--XI sections). In this paper, we propose non-classical adaptive wavelet expansions with $\varepsilon$ - compression.

In this article, the following results were obtained. The number of arithmetic operations in obtaining the proposed wavelet decomposition is of the same order as the number of operations required to $\varepsilon$-compression of the initial flow. If optimal $\varepsilon$-compression is needed, then in general $O\left(M^{2}\right)$ arithmetic operations are required (see XII Section). If it is known that the initial flow has the convexity property, then for optimal $\varepsilon$-compression it is possible to use only $O\left(M \log _{2} M\right)$ arithmetic operations (see XIII Section). If the initial flow is a flow of values of a function and its derivative, then adaptive compression can be obtained using $O(M)$ arithmetic operations (see II -- IV sections). It is possible to replace the values of the derivative with difference relations and at the same time also obtain compression using $O(M)$ arithmetic operations (when replacing the derivatives with the simplest difference relations, the number of arithmetic operations increases by about four times).

\section{References}

[1] Haar A. "Zur Theorie der orthogonalen Funktionensysteme", Mathematische Annalen, 69, pp. 331-371, 1910. https://link.springer.com/article/10.1007/BF01456326.

[2] Kazuo Toraichi, Kazuki Katagishi, Iwao Sekita \&Ryoichi Mori. Computational complexity of spline interpolation// International Journal of Systems Science,Volume 18, 1987 Issue 5, pages 945-954.

[3] Friedman, J.H. Multivariate adaptive regression splines, The Annals of Statistics, 19, 1 (1991) 1-141.

[4] Michael Unser, Akram Aldrobi, and Murray Eden. FastvBSpline Transforms for Continuous Image Representation and Interpolation //IEEE Transactions on Pattern Analysis and Machine Intellegence, Vol.13, No. 3, March 1991, 277-285.

[5] Cai, W. and Wang, J., "Adaptive Multiresolution Collocation Methods for Initial Boundary Value Problems of Nonlinear PDEs", SIAM J. Num. An., vol. 33, iss. 3, pp. $937-$ 970, 1996. https://www.jstor.org/stable/2158490?seq=1

[6] S.G. Michlin, Approximation auf dem Kubischen Gitter, Berlin, 1970. ISBN-s: 3034854994 / 9783034854993.

[7] B.K. Swartz, and R.S. Varga, "Error bounds for spline and L-spline interpolation", J. Approximation 6, pp. 6-49, 1972. 
[8] L.L. Schumaker, "Constructive aspects of discrete polynomial spline functions", Approximation Theory, G.G. Lorentz, ed., Academic Press, New York, pp. 469-476, 1973.

[9] S. Mallat, A Wavelet Tour of Signal Processing, Academic Press, 1999. DOI: 10.2118/96553-MS.

[10] Francois Dubeau, Said Elmejdani, Riadh Ksantini.

"Non-uniform Haar wavelets", Applied Mathematics and Computation. 159, 675-691, 2004.

https://www.sciencedirect.com/science/article

/abs/pii/S009630030301155X

[11] Ivo Babuska, Uday Banerjee, John E. Osborn, "Generalized Finite Element Methods: Main Ideas, Results, and Perspective", International Journal of Computational Methods 1 (1), pp.67-103, 2004. DOI:

10.1142/S0219876204000083.

[12] O.C. Zienkiewicz, R.L. Taylor, J.Z. Zhu, The Finite Element Method: Its Basis and Fundamentals (Sixth ed.). Butterworth-Heinemann. 2005. ISBN 0-750-6320-0.

[13] J.N. Reddy, An Introduction to the Finite Element Method (Third ed.). McGraw-Hill. 2006.

ISBN 0-07-051355-4.

[14] K.J. Bathe, Finite Element Procedures, Cambridge, MA: Klaus-Jurgen Bathe, 2006. ISBN 978-0-9790049-5-7.

[15] G. R. Liu, T. T. Nguyen, K. Y. Dai and K. Y. Lam, "Theoretical aspects of the smoothed finite element method (SFEM)”, Int. J. Numer. Meth. Engng. 71, pp.902-930, 2007. DOI: $10.1002 /$ nme. 1968.

[16] G. R. Liu, K. Y. Dai, T. T. Nguyen, "A smoothed finite element method for mechanics problems", Comput. Mech. 39, pp.859 - 877, 2007. DOI: 10.1007/s00466-006-0075-4

[17] Yu. K. Demyanovich, Wavelet decompositions on nonuniform grids Am. Math. Soc. Transl. Ser. 2, 222, pp.2342, 2008. DOI: 10.1007/s10958009-9752-0.

[18] G.R. Liu, G.R. Zhang, "Edge-based Smoothed Point Interpolation Methods", International Journal of Computational Methods, 5(4), 2008, pp.621-646. DOI: 10.1142/S0219876208001510.

[19] Yu. K. Demyanovich, Wavelets on a manifold, Dokl. Math. 79, No. 1, pp.2124, 2009. DOI:10.1134/S1064562409010074

[20] Z.Q. Zhang, G.R. Liu, "Upper and lower bounds for natural frequencies: A property of the smoothed finite element methods", International Journal for Numerical Methods in Engineering, 84, Issue: 2, pp.149-178 2010. DOI: 10.1016/S0894-9166(13)60014-2
[21] G.R. Liu, Smoothed Finite Element Methods, CRC Press, 2010. ISBN 9781439820278.

[22] T. Nguyen-Thoi, G. R. Liu and H. Nguyen-Xuan, "An nsided polygonal edge-based smoothed finite element method (NES-FEM) for solid mechanics", Int. J. Numer. Meth. Biomed. Engng. 2010, (www.interscience.wiley.com). DOI: 10.1002/cnm.1375.

[23] Novikov I.Ya., Protasov V.Yu., Skopina M.A. Wavelet Theory. AMS, Translations Mathematical Monographs, V. 239, 2011. ISBN 978-08218-4984-2.

[24] Eric Nyiri, Olivier Gibaru, Philippe Auquiert. Fast L1-Ck polynomial spline interpolation algorithm withshapepreservingproperties// Computer Aided Geometric Design, Elsevier, 2011, 28(1), pp.65-74.

[25] Mehra, M. and Goyal, K., "Algorithm 929: A Suite on Wavelet Differentiation Algorithms", ACM Transact. Math. Soft., vol. 39, iss. 4, art. no. 27, 2013. http://web.iitd.ac.in/ mmehra/publication/ACM.pdf

[26] B. I. Kvasov, "Monotone and convex interpolation by weighted cubic splines", Comput. Math. Math. Phys., 53:10, pp. 1428-1439, 2013.

[27] I.G. Burova, T.O. Evdokimova, "On construction third order approximation using values of integrals", WSEAS Transactions on Mathematics, 13, pp. 676-683, 2014.

[28] M. Scarpiniti, D. Comminiello, R. Parisi, and A. Uncini, "Hammerstein uniform cubic spline adaptive filters: Learning and convergence properties," //Signal Processing, vol. 100, pp. 112-123, 2014.

[29] I.G.Burova, Yu.K.Dem'yanovich, "On properties of Decomposition Operations for Spline-Wavelet Representations", J. of Math. Science, 205, 2, pp.205-221, 2015.

[30] Yu. K. Demyanovich and A.Yu.Ponomareva. "Adaptive Spline-Wavelet Processing of a Discrete Flow", J. Math. Sci., New York 210, No 4, pp.371-390, 2015. DOI: 10.1007/s10958-015-2571-6.

[31] Vahid Shobeiri, "Structural Topology Optimization Based on the Smoothed Finite Element Method", Latin American Journal of Solids and Structures, 13, pp.378-390, 2016. DOI: $10.1590 / 1679-78252243$.

[32] W. Zeng, G.R. Liu, "Smoothed finite element methods (S-FEM): An overview and recent developments", Archives of 
Computational, Methods in Engineering, 2016. DOI: 10.1007/s11831-016-9202-3.

[33] Shumilov, B.M., Algorithms with Splitting for the Wavelet Transform of Splines of the First Degree on Nonuniform Grids, Zh. Vych. Mat. Mat. Fiz., vol. 56, no. 7, pp. 39-50, 2016.

https://link.springer.com/article/10.1134/S199542391701 $\underline{0098}$

[34] Xiaogang Du, Jianwu Dang, Yangping Wang, Song Wang, and Tao Lei. A Parallel Nonrigid Registration Algorithm Based on B-Spline for Medical Images//Computational and Mathematical Methods in Medicine Volume, 2016, Article ID 7419307, 14 pages http://dx.doi.org/10.1155/2016/7419307

[35] Neira J, Reid I. Leonard JJ. Past, present, and future of simultaneous localization and mapping: Toward the robustperception age. IEEE Transactions on Robotics, 32(6), pp. 1309-1332, 2016.

[36] I.G. Burova, O.V. Rodnikova, "Integro-differential polynomial and trigonometrical splines and quadrature formulae", WSEAS Transactions on Mathematics, 16, pp. 11$18,2017$.

[37] Yu.K.Dem'yanovich, A.S.Ponomarev. "On realization of the SplineWavelet Decomposition of the First Order", J. of Math. Sci. Vol. 224, No.6, pp.833-860. 2017. DOI: 10.1007/s10958-017-3454-9.

[38] Yuri K. Demyanovich, Tatjana O. Evdokimova, Evelina V. Prozorova, "On General Smoothness of Minimal Splines of the Lagrange Type", WSEAS TRANSACTIONS on MATHEMATICS, pp. 304-310, Volume 17, 2018.

[39] Yuri K. Demyanovich,"Parallelizationo Spline-Wavelet Decompo-sition", WSEAS TRANSACTIONS on MATHEMATICS, pp. 241-249, Volume 18, 2019.

[40] Maple 2017.0, Product Build IDs, Maple Build ID 1231047, Licensed to: Prof. Yuri Demyanovich, Serial Number: M4SUJR24AKMC7YDY, Permanent Licence.

\section{Creative Commons Attribution License 4.0 (Attribution 4.0 International, CC BY 4.0)}

This article is published under the terms of the Creative Commons Attribution License 4.0

https://creativecommons.org/licenses/by/4.0/deed.en US 